This is an open access article distributed under the terms of the Creative Commons BY-NC-ND Licence

\title{
Overexpression of a gene $A h F B A$ from Arachis hypogaea confers salinity stress tolerance in Escherichia coli and tobacco
}

\author{
Z.K. DU ${ }^{1,2}$,Y.F. HU ${ }^{3}$, and J.M. LI ${ }^{1,2 *}$ \\ Zhejiang Provincial Key Laboratory of Plant Evolution and Conservation, Taizhou University, \\ Taizhou, Zhejiang 318000, P.R. China ${ }^{1}$ \\ Institute of Ecology, Taizhou University, Taizhou, Zhejiang 318000, P.R. China ${ }^{2}$ \\ College of Agronomy, Sichuan Agricultural University, Chengdu, Sichuan 611130, P.R. China ${ }^{3}$
}

\begin{abstract}
Fructose-1,6-bisphosphate aldolase (FBA), an essential enzyme involved in the glycolytic pathway, gluconeogenesis, and the Calvin cycle, plays significant roles in the regulation of plant growth, development, and stress responses. In this study, a novel gene, AhFBA (GenBank accession number KF470788), containing a 1077-bp open reading frame and encoding a protein of 358 amino acids, was isolated from Arachis hypogaea L. Bioinformatic analysis revealed that AhFBA belonged to class-I aldolases and preferentially localized in the cytoplasm. Real-time quantitative PCR analysis indicated that $A h F B A$ had a higher expression in young fruits than in leaves and stems, and $\mathrm{NaCl}$ could trigger the highest expression of $A h F B A$ in roots and leaves after 3-h and 6-h treatments. The salinity tolerance and survival of Escherichia coli transformed with $A h F B A$ were notably enhanced compared with the control. Transgenic tobacco (Nicotiana tabacum L.) overexpressing the AhFBA gene exhibited a lower hydrogen peroxide content, electrolyte leakage, and malondialdehyde content and a higher photosynthetic efficiency, net photosynthetic rate, relative water content, and sucrose and proline content compared with control plants. Taken together, the results demonstrate that $A h F B A$ functioned as a positive factor enhancing the tolerance of $E$. coli and $N$. tabacum to salinity stress, possibly by maintaining the osmotic balance and scavenging hydrogen peroxide.
\end{abstract}

Additional key words: fructose-1,6-bisphosphate aldolase, $\mathrm{H}_{2} \mathrm{O}_{2}$, net photosynthetic rate, Nicotiana tabaccum, peanut, proline, RWC.

\section{Introduction}

Fructose-1,6-bisphosphate aldolase (FBA, EC 4.1.2.13) catalyzes the reversible reaction of aldol fructose-1,6bisphosphate (FBP) into triose dihydroxyacetone phosphate (DHAP) and glyceraldehyde-3-phosphate (G3P), which is a key enzyme for glycolysis, gluconeogenesis, and the Calvin cycle (Haake et al. 1998). The FBAs can be divided into two groups according to the catalytic mechanism employed. Class I FBAs are mainly found in plants, animals, and green algae (only occasionally in bacteria), forming a Schiff-base using the $\varepsilon$-amino group of a conserved lysine residue in the active center of the enzyme with the C-2 carbonyl group of the substrate (FBP or DHAP) (Shams et al. 2014). Class I FBAs can form tetramers in eukaryotes, and they are not inhibited by a chelating agent EDTA. However, class II FBAs are mainly found in bacteria and fungi, utilizing a divalent metal ion (usually $\mathrm{Zn}^{2+}$ or $\mathrm{Fe}^{2+}$ ) at the active site as an electrophile to stabilize the carbanion formed on the third carbon of the substrate. Furthermore, class II FBAs are dimers and can be inhibited by EDTA (Gross et al. 1999).

Submitted 2 March 2018, last revision 21 June 2018, accepted 7 August 2018.

Abbreviations: DHAP - dihydroxyacetone phosphate; EC - electrical conductivity; FBA - fructose-1,6-bisphosphate aldolase; FBP - fructose-1,6-bisphosphate; G3P - glyceraldehyde-3-phosphate; IPTG - isopropyl $\beta$-D-1-thiogalactopyranoside; LB - LuriaBertani; MDA - malondialdehyde; ORF - open reading frame; qPCR - quantitative polymerase chain reaction; $\mathrm{PN}_{\mathrm{N}}$ - net photosynthetic rate; ROS - reactive oxygen species; RWC - relative water content.

Acknowledgments: This study was funded by the Public Welfare Project of Science Technology Department of Zhejiang Province (LGN18C160002), the National Natural Science Foundation of China (31270461; 31571757), and the Science and Technology Program of Yuhuan City, China (201603).

* Corresponding author; fax: (+86) 576 88660892; e-mail: lijm@tzc.edu.cn 
The FBAs in green plants have two isozymes, a cytosolic FBA (cFBA) and a chloroplast/plastid FBA (cpFBA) (Lebherz et al. 1984). The cFBA is a vital enzyme that catalyzes the reversible cleavage of FBP into DHAP and GAP in the glycolytic and gluconeogenesis pathways in the cytoplasm, which plays an important role in plant growth and tolerance to environmental stresses. Antisense inhibition of OscFBA expression in rice decreases the amount of FBA in roots and suppresses the growth of roots compared with wild-type plants (Konishi et al. 2004). Likewise, cFBA is more active in elongating tissues of Phyllostachys pubescens than in those that have completed elongation (Lao et al. 2013). Moreover, overexpression of CSIFBA in tomato enhances the seed germination rate under low and high temperature stresses (Cai et al. 2016). In contrast, cpFBA is an essential enzyme in the Calvin cycle, where it catalyzes the reversible reaction of sedoheptulose-1,7-bisphosphate into DHAP and erythrose-4-phosphate in the chloroplast, in addition to the reversible condensation of DHAP and G3P to FBP (Flechner et al. 1999). Overexpression of the Arabidopsis thaliana cpFBA gene in tobacco significantly enhances the photosynthesis $\mathrm{CO}_{2}$ fixation rate and growth of transgenic plants, particularly at a high $\mathrm{CO}_{2}$ concentration (Uematsu et al. 2012).

Increasing evidence has suggested that FBA genes participate in many physiological and biochemical reactions in plants, including chloroplast development (Zhang et al. 2016), $\mathrm{CO}_{2}$ fixation (Uematsu et al. 2012), plant growth (Lao et al. 2013), and oil yield (Zeng et al. 2014), as well as in plant resistance to biotic (Mohapatra and Mittra 2016) and abiotic stresses such as drought (Cheng et al. 2016), chilling (Purev et al. 2008), salt (Zeng et al. 2015), and cadmium (Singh et al. 2015). A large number of FBA genes have been identified in many plant species, e.g., in maize (Dennis et al. 1988), rice (Kagaya et al. 1995), Camellia oleifera (Zeng et al. 2015), tomato (Cai et al. 2016), and wheat (Lv et al. 2017).

Although studies on FBA genes from other species have been widely reported, little is known about this gene in peanut. Peanut, as a main source of vegetable oil and plant protein, is widely planted in tropical and subtropical regions, of which at least $15 \%$ are affected by salt stress (Kavas et al. 2015). Therefore, to achieve a better understanding of the molecular mechanism of salt tolerance in peanut, it is necessary to identify the responses of its genes to salinity stress. A recent report showed that the FBA gene of $A$. hypogaea is responsive to salinity stress (Sui et al. 2016); however, the functions of this gene under salt stress are still not clear. Therefore, a novel cytoplasmic gene $A h F B A$ was isolated from peanut and used to generate $A h F B A$-overexpressing Escherichia coli and tobacco lines to determine the physiological functions of AhFBA.

\section{Materials and methods}

Plants and growth conditions: Peanut (Arachis hypogaea L. cv. Xiaojingsheng) seeds purchased from the Xinchang Seeds Company were sterilized by soaking in a $2 \%(\mathrm{~m} / \mathrm{v})$ $\mathrm{NaClO}_{4}$ for $10 \mathrm{~min}$ and grown in a growth chamber at a temperature of $25^{\circ} \mathrm{C}$, a relative humidity of $75 \%$, a $16-\mathrm{h}$ photoperiod, and an irradiance of $400 \mu \mathrm{mol} \mathrm{m}^{-2} \mathrm{~s}^{-1}$ until the fourth leaf was fully expanded. Seeds of Nicotiana tabaccum L. cv. Yunan 85 were surface-sterilized with $70 \%(\mathrm{v} / \mathrm{v})$ ethanol for $2 \mathrm{~min}$ and $1 \%(\mathrm{~m} / \mathrm{v}) \mathrm{NaClO}_{4}$ for $10 \mathrm{~min}$ followed by six washes with sterile water. They were then sown in plastic trays filled with a mixture of Perlite and Vermiculite $(1: 1, \mathrm{v} / \mathrm{v})$ for germination in the above mentioned growth chamber.

Isolation of RNA and cDNA synthesis: Total RNA was extracted from peanut leaf samples with a TRIzol reagent (Promega, Madison, USA) according to the manufacturer's instructions. An RNA quality assessment was performed by agarose gel electrophoresis, and the concentration was measured with a NanoDrop ${ }^{\mathrm{TM}} N D-1000$ (Nanodrop Technologies, Wilmington, DE, USA) spectrophotometer. The first-strand cDNA was synthesized from total RNA using a PrimeScript ${ }^{\mathrm{TM}} 1 \mathrm{st}$ strand synthesis kit (TaKaRa, Dalian, China) according to the manufacturer's instructions.
In silico cloning the AhFBA gene from Arachis hypogaea: The protein sequence of FBA (GenBank accession number AY492006.1) from Glycine max was used as a query probe for a $t B L A S T n$ search against the Arachis hypogaea EST database at the National Center for Biotechnology Information (NCBI) website. A total of 15 peanut ESTs (GO257556.1, FS966168.1, FS971324.1, FS961748.1, FS982176.1, FS965569.1, FS979209.1, FS969910.1, ES705591.1, FS974377.1, FS978710.1, ES710499.1, FS973585.1, FS766474.1, and FS979097.1) sharing a high similarity with the reference sequence were subjected to contig assembly using the SeqMan program, and an EST contig with a length of 1376 bp was obtained. The putative full-length cDNA of AhFBA was analyzed using ORF Finder, and one open reading frame (ORF) with a length of 1077 bp was identified. Primers AhFBAC-F and AhFBA-C-R (Table 1 Suppl.) were then designed to amplify the putative ORF sequence using cDNA as a template.

Experimental verification and sequence analyses of AhFBA: Reaction mixtures for PCR $\left(20 \mathrm{~mm}^{3}\right)$ contained $2.0 \mathrm{~mm}^{3}$ of template cDNA $(20 \mathrm{ng}), 2.5 \mathrm{~mm}^{3}$ of dNTP mixture_(2.5 mM dATP, $2.5 \mathrm{mM}$ dGTP, $2.5 \mathrm{mM} \mathrm{dCTP}$, and $2.5 \mathrm{mM}$ dTTP), $2.0 \mathrm{~mm}^{3}$ of $10 \times$ PCR buffer, $0.1 \mathrm{~mm}^{3}$ of each primer $(10 \mu \mathrm{M}), 0.1 \mathrm{~mm}^{3}$ of KOD plus DNA 
polymerase (Toyobo, Shanghai, China), and $13.2 \mathrm{~mm}^{3}$ of double distilled $\mathrm{H}_{2} \mathrm{O}$. The PCR reaction program was as follows: an initial denaturation at $94{ }^{\circ} \mathrm{C}$ for 5 min followed by 30 cycles of denaturation at $94^{\circ} \mathrm{C}$ for $40 \mathrm{~s}$, annealing at $55^{\circ} \mathrm{C}$ for $40 \mathrm{~s}$, and extension at $72{ }^{\circ} \mathrm{C}$ for $1.5 \mathrm{~min}$, and a final extension reaction at $72{ }^{\circ} \mathrm{C}$ for $10 \mathrm{~min}$. The PCR products were detected on a $1 \%(\mathrm{~m} / \mathrm{v})$ agarose gel, and then the single specific band was purified using a DNA gel extraction kit (Beyotime, Haimen, China) and ligated to the pUCm-T vector (Beyotime) to construct a recombinant vector pUCm-T-AhFBA. Positive clones were sequenced with an ABI 3130XL genetic analyzer (Applied Biosystems, Foster City, USA).

Bioinformatics analyses: Amino acid sequence alignment analysis was performed with DNAMAN 7.0 (Lynnon Biosoft, Quebec, Canada). The protein active site was identified by searching the Motif Scan database (http://myhits.isb-sib.ch/cgi-bin/motif_scan). Signal peptides were predicted using SignalP 4.1 (http:// www.cbs.dtu.dk/services/SignalP-4.1/). A phylo-genetic tree of FBA proteins in different species was constructed with MEGA 5.05 (Tempe, AZ, USA) using the unweighted pair-group method with the arithmetic mean. The AhFBA protein sequence (GenBank accession number AGV08373.1) was submitted to SwissModel (http:// swissmodel.expasy.org/) for structural modeling. The modeled tertiary and quaternary structures were analyzed in Swiss-PdbViewer.

Gene expression profile analysis by real-time quantitative PCR: A pair of specific primers, AhFBASYBR-F and AhFBA-SYBR-R (Table 1 Suppl.), avoiding the conserved region, were designed for real-time quantitative PCR (qPCR) to amplify a product of $100-200$ bp from AhFBA cDNA of roots, leves, flowers, gynophores, and young fruits. The $18 \mathrm{~S}$ rRNA gene was used as an internal control gene. Cycle threshold $(\mathrm{Ct})$ values were determined for each sample, and the relative gene expression levels of control gene expression were calculated using the $2^{-\Delta \Delta \mathrm{Ct}}$ method (Schmittgen and Livak 2008).

Expression of a recombinant protein AhFBA in E. coli: The PCR products of the AhFBA gene amplified with primers AhFBA-E-F and AhFBA-E-R (Table 1 Suppl.) were purified with a DNA gel extraction kit (Beyotime) and digested with BamHI and SalI followed by ligation into pET28a $(+)$ digested with the same restriction endonucleases to construct a recombinant plasmid pET28a-AhFBA. The plasmid pET28a-AhFBA was then transformed into $E$. coli strain BL21(DE3), and positive clones were verified by sequencing. The positive recombinant cells were cultivated at $37{ }^{\circ} \mathrm{C}$ in a LuriaBertani (LB) medium containing $50 \mathrm{mg} \cdot \mathrm{dm}^{-3}$ kanamycin until the cells were grown to the mid-log phase $\left(\mathrm{A}_{600} \approx 0.5\right)$, followed by induction with $0.1 \mathrm{mM}$ isopropyl- $\beta-\mathrm{D}-1$ - thiogalacto-pyranoside (IPTG) at $37{ }^{\circ} \mathrm{C}$ on a shaking incubator at $120 \mathrm{rpm}$ for $1,2,3,4$, and $5 \mathrm{~h}$. The soluble protein fractions were separated on a $12 \%(\mathrm{~m} / \mathrm{v})$ SDS-PAGE gel and stained with Coomassie Brilliant Blue G-250.

Assay for salt tolerance of $E$. coli BL21(DE3) transformed with pET28a-AhFBA: Single colonies of BL21(DE3) cells harboring either pET28a or pET28aAhFBA were inoculated on a LB liquid medium containing $50 \mathrm{mg} \cdot \mathrm{dm}^{-3}$ kanamycin. Induction of AhFBA protein was performed according to the procedure described above using $0.1 \mathrm{mM}$ IPTG for $2 \mathrm{~h}$. The cultures were serially diluted 10 -fold, and then $5-\mathrm{mm}^{3}$ dilutions of each sample were spotted onto LB medium plates containing $0.1 \mathrm{mM}$ IPTG and $200 \mathrm{mM} \mathrm{NaCl}$. The bacteria were grown in the dark at $37^{\circ} \mathrm{C}$ for $12 \mathrm{~h}$ and observed with a digital camera. In other experiment, cultures of BL21(DE3) (harboring pET28a or pET28a-AhFBA) were inoculated on $50 \mathrm{~cm}^{3}$ of a LB medium supplemented with $50 \mathrm{mg} \cdot \mathrm{dm}^{-3}$ kanamycin and $200 \mathrm{mM} \mathrm{NaCl}$. Absorbance at $600 \mathrm{~nm}$ of the bacterial cultures were adjusted to approximately 0.2 and induced by $0.1 \mathrm{mM} \mathrm{IPTG}$ at $37^{\circ} \mathrm{C}$. Samples of $1 \mathrm{~cm}^{3}$ were harvested every $60 \mathrm{~min}$, and absorbance at $600 \mathrm{~nm}$ was measured using a T6 spectrophotometer (Puxi, Shanghai, China).

Subcellular localization of AhFBA protein: The ORF of AhFBA was amplified using specific primers AhFBA-E-F and AhFBA-E-R (Table 1 Suppl.), and then the purified PCR product was digested with BamHI and SalI followed by insertion into the multiple cloning site of the expression vector pCAMBIA1300-GFP to generate a recombinant vector pCAMBIA1300-AhFBA-GFP. The vector pCAMBIA1300-AhFBA-GFP was introduced into Nicotiana tabacum cv. Yunyan 85 cells via Agrobacterium tumefaciens LBA4404 (Zhang et al. 2015), and the AhFBA-GFP fusion protein was induced under the control of the CaMV 35S promoter from the cauliflower mosaic virus. The green fluorescence protein signal was observed with a confocal laser scanning fluorescence microscope (Olympus, Tokyo, Japan).

Transformation of AhFBA in tobacco: LBA4404 harboring the recombinant plasmid pCAMBIA1300AhFBA-GFP was introduced into N. tabacum cv. Yunyan 85 leaves by the leaf-disc method. Putative transgenic seedlings were screened on Murashige-Skoog (MS) medium plates containing $100 \mathrm{mg} \cdot \mathrm{dm}^{-3}$ kanamycin and confirmed by PCR analysis using primers AhFBA-E-F and AhFBA-E-R (Table 1 Suppl.). The seeds of all transgenic lines were harvested and germinated on an MS solid medium containing $100 \mathrm{mg} \cdot \mathrm{dm}^{-3}$ kanamycin to select transgenic lines carrying a single copy of the integrated AhFBA gene, and transgenic offspring showing the expected segregation ratio of 3:1 were chosen for the production of $\mathrm{T}_{1}$ progeny. 
Salinity tolerance of transgenic tobacco: Seeds from $T_{1}$ progeny transgenic lines were surface-sterilized with $70 \%$ $(\mathrm{v} / \mathrm{v})$ ethanol for $2 \mathrm{~min}$ and $1 \%(\mathrm{~m} / \mathrm{v}) \mathrm{NaClO}_{4}$ for $10 \mathrm{~min}$ followed by six washes with sterile water. They were then sown in plastic trays filled with a mixture of Perlite and Vermiculite $(1: 1, \mathrm{v} / \mathrm{v})$ for germination in the above mentioned growth chamber. Two-week-old control (transformed with the empty vector pCAMBIA1300-GFP) and $T_{1}$ transgenic seedlings were transplanted into pots containing soil, humus, and sand $(2: 1: 1, \mathrm{v} / \mathrm{v} / \mathrm{v})$ and then cultured for three weeks. To evaluate salt tolerance, transgenic and control plants were irrigated with $500 \mathrm{mM}$ $\mathrm{NaCl}$ every third day for $6 \mathrm{~d}$. After $\mathrm{NaCl}$ treatment, the third and fourth leaves from the shoot apex were used for measurements of gas exchange parameters and physiological analyses.

Measurements of physiological parameters: Chlorophyll fluorescence was measured at $25{ }^{\circ} \mathrm{C}$ using a OS30P portable modulated fluorometer (Opti-Sciences, Hudson, NH, USA) as described by Toscano et al. (2016). Net photosynthesis rate $\left(\mathrm{P}_{\mathrm{N}}\right)$ was measured using a LI-6400 portable photosynthesis system ( $\mathrm{Li}$-Cor, Lincoln, USA). The parameters in the measuring chamber were: a
$\mathrm{CO}_{2}$ concentration of $400 \mu \mathrm{mol} \cdot \mathrm{mol}^{-1}$, photosynthetically active radiation $(\mathrm{PAR})$ of $1500 \mu \mathrm{mol} \cdot \mathrm{m}^{-2} \cdot \mathrm{s}-1$, a temperature of $25^{\circ} \mathrm{C}$ and a relative humidity of $65 \%$.

Determination of the relative water content (RWC) and proline content were performed according to Patel et al. (2015) by gravimetric method and acidic ninhydrin method, respectively. Content of $\mathrm{H}_{2} \mathrm{O}_{2}$ was assessed with potassium iodide (KI) reagent following Chen et al. (2016). Content of MDA and electrolyte leakage were determined according to Park et al. (2017) by thiobarbituric acid (TBA) method and electrical conductivity method, respectively. Sucrose content was measured by adding hydroxyphenol solution and recording absorbance at $480 \mathrm{~nm}$ described by Shi et al. (2012).

Statistical analysis: Statistical analyses were performed using three independent biological replicates. The values provided in the figures and tables are presented as means \pm SEs. Data were analyzed by one-way analysis of variance (ANOVA) and the Duncan multiple comparison test using the SPSS 18.0 software (SPSS, Chicago, IL, USA). Different lowercase letters on the histograms indicate that the means were significantly different at $P<0.05$.

\section{Results}

Two primers were employed to amplify the ORF of AhFBA, and one approximately $1000-\mathrm{bp}$ real-time PCR product was harvested (Fig. 1 Suppl.) and sequenced in both directions. Sequence comparisons between the assembled contig and the PCR product showed that the two sequences were the same, supporting the credibility of the in silico cloning results. The AhFBA cDNA generated by PCR contained an ORF of 1077 bp encoding 358 amino acid residues, and the sequence was submitted to the GenBank (accession number KF470788.1). The deduced AhFBA protein had a predicted molecular mass of 38.38 $\mathrm{kDa}$ and a theoretical isoelectric point of 6.73. Signal peptide prediction by SignalP revealed no signal peptide in the protein of AhFBA, implying that AhFBA might not be a secreted protein.

Multiple sequence alignment of the amino acid sequences showed that the sequence of AhFBA protein was highly homologous to that of other plant FBA sequences, sharing 92.18, 91.34, 91.06, 89.66, 87.43, 85.75 , and $84.36 \%$ amino acid sequence similarity with Medicago truncatula, Phaseolus vulgaris, Glycine soja, Citrus clementina, Arabidopsis lyrata, Solanum tuberosum, and Triticum aestivum, respectively. Furthermore, it also contained one conserved domain, VLLEGTLLKPN (217-227), the class-I active site of FBA. This conserved motif could form a Schiff-base between the $\varepsilon$-amino group of lysine in the active site and the carbonyl group of the substrate (Fig. 2 Suppl.).

To study the phylogenetic relationship of AhFBA proteins from a variety of plants, a phylogenetic tree was constructed and revealed three major clades, dicotyledons, monocotyledons, and green algae (Fig. 3 Suppl.). AhFBA was grouped into one cluster of dicotyledons together with FBAs from other legume plants including M. truncatula, P. vulgaris and G. soja (Fig. 3 Suppl.).

The tertiary and quaternary structures of the AhFBA protein were predicted with SwissModel. The results showed that the monomer of AhFBA consisted of $12 \alpha$-helix and $12 \beta$-sheet secondary structures (Fig. 1A). In addition, AhFBA could be classified as a class I subfamily because it could form homotetramers containing interfaces $\mathrm{A}$ and $\mathrm{B}$, similar to other class I subfamily FBAs. In AhFBA, each interface B had six hydrogen bonds consisting of the carboxylate $\mathrm{O}$ atoms of Asp124 and the backbone amides of three consecutive residues (G122-L123-D124) of the homologous subunit. Compared with interface $\mathrm{B}$, which displayed loop-loop interactions formed by hydrogen bonds, interface A was composed of helix-packing interactions (Fig. 1B). Although the peripheral amino acid residues of the active site differed among the FBAs, the catalytic residues in the active site interior were highly conserved. The catalytic residues in AhFBA had also highly conserved catalytic residues, which included D30-K103-K142-R144-E183-E185K225-S266-R298 (Fig. 1C).

To analyze the expression patterns of AhFBA, mRNA isolated from different tissues of peanut was used as the template for real-time qPCR. Although the AhFBA gene 
transcription was detected in all tested tissues, the expressions were significantly different $(P<0.05)$. The transcript abundances of AhFBA in leaves were lowest, and those in young fruits, gynophores, and roots were approximately 4.0-, 5.3- and 8.3-fold higher, respectively (Fig. 2A). To characterize the expression pattern of the $A h F B A$ gene under $\mathrm{NaCl}$ stress for various times, real-time
qPCR was also employed. As shown in Fig. $2 B$, the transcription of $A h F B A$ was upregulated and showed approximately 7.4- and 5.8-fold increases at 3 and $6 \mathrm{~h}$ after the treatment with $400 \mathrm{mM} \mathrm{NaCl}$ in roots and leaves, respectively. These results suggest that the AhFBA gene could be induced by $\mathrm{NaCl}$ treatment.
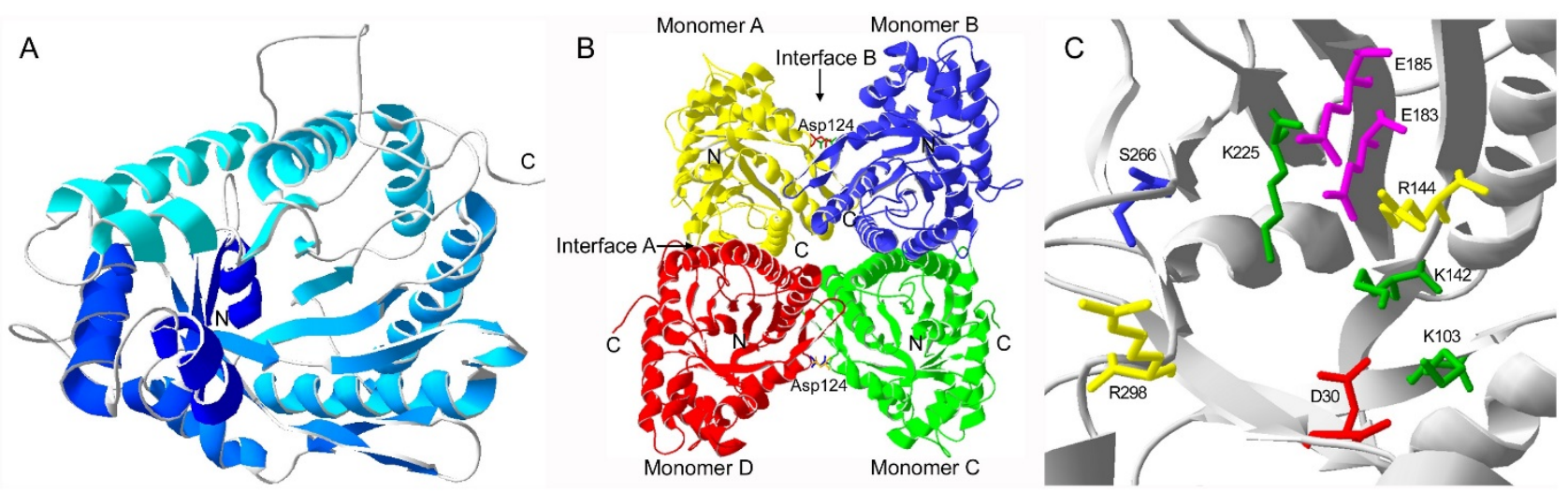

Fig. 1. Predicted tertiary and quaternary structures of the AhFBA protein. $A$ - Monomer AhFBA; $B$ - tetrameric AhFBA; $C$ - amino acid residues in the active site of AhFBA. For $A, B$, carboxyl-termini and amino-termini are denoted by $C$ and $N$, respectively. For $B$, each monomer in the tetramer is labeled with one color, and the arrows indicate interfaces A and B. The Asp124 forms hydrogen bonds with amino acid residues in the adjacent monomer on interface B.

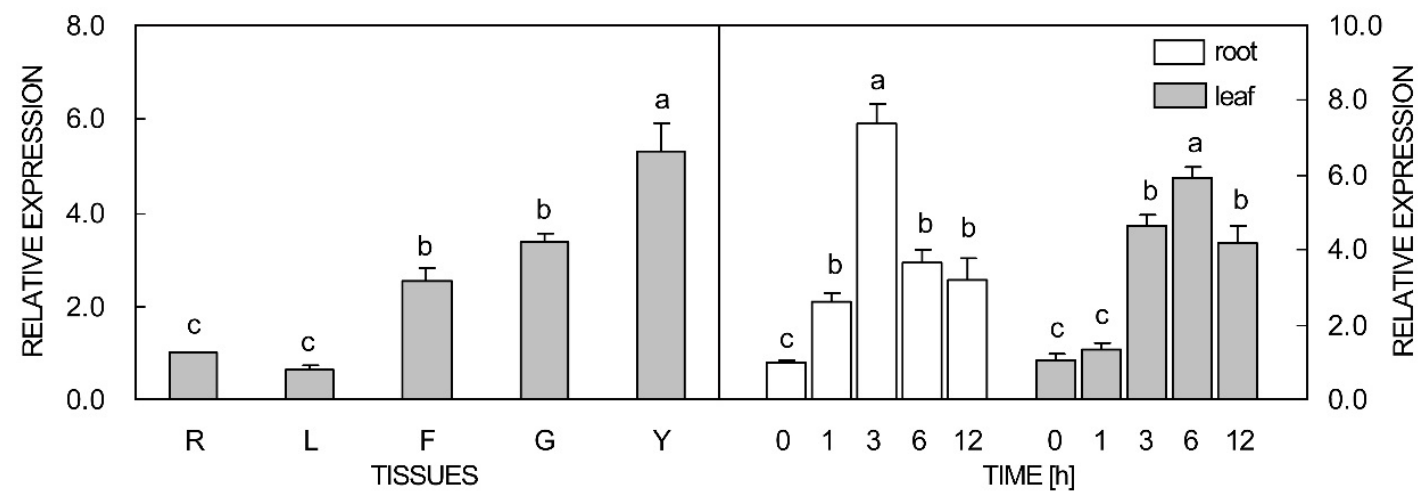

Fig. 2. Relative expressions of AhFBA in different tissues of Arachis hypogaea measured by real-time quantitative PCR. Expression patterns of $A h F B A$ in roots (R), leaves (L), flowers (F), gynophores $(\mathrm{G})$, and young fruits (Y) of control plants (on the left). Expression patterns of $A h F B A$ roots and leaves under $400 \mathrm{mM} \mathrm{NaCl}$ for 1 to $12 \mathrm{~h}$ (on the right). Data are the means $\pm \mathrm{SEs}$ from three biological replicates. Different lowercase letters indicate significant differences $(P<0.05)$.

The recombinant AhFBA protein was strongly induced by IPTG in E. coli strain BL21(DE3) when the coding sequence of $A h F B A$ was integrated into the multiple cloning site of the expression vector $\mathrm{pET} 28 \mathrm{a}$ under control of the T7 promoter. A distinct protein band with a molecular mass between 31.0 and $43.0 \mathrm{kDa}$, consistent with the predicted value, was detected on the SDS-PAGE gel of total proteins following induction for 1 to $5 \mathrm{~h}$ with $0.1 \mathrm{mM}$ IPTG, compared to the BL21(DE3) strain containing pET28a incubated with IPTG (Fig. 3A).

To assess whether AhFBA is involved in protecting against $\mathrm{NaCl}$ stress, a spotting assay was employed to investigate the growth response of $E$. coli cultures (with and without expression of recombinant AhFBA) under $\mathrm{NaCl}$ stress. The plate spotting experiments revealed that both pET28a-AhFBA and pET28a (control) vectortransformed E. coli BL21(DE3) cells had similar growth performances (Fig. 3B). However, pET28a-AhFBAtransformed cells showed a significant growth advantage (a larger size and greater number of colonies) compared with control cells when subjected to the addition of 200 $\mathrm{mM} \mathrm{NaCl}$ to the LB medium (Fig. 3C). In the liquid medium, pET28a-AhFBA-bearing E. coli cells entered a logarithmic phase earlier than pET28a-bearing E. coli cells, but absorbance at $600 \mathrm{~nm}$ did not differ between them after cultivation for $11 \mathrm{~h}$ in the absence of $\mathrm{NaCl}$ 
(Fig. 3D). Addition of $200 \mathrm{mM} \mathrm{NaCl}$ to the LB medium inhibited the growth of both cell strains, but $E$. coli cells harboring pET28a-AhFBA showed a significantly diminished reduction of the growth rate compared with E. coli cells harboring pET28a (Fig. 3E).

To explore how the function of $A h F B A$ is related to $\mathrm{NaCl}$ stress adjustment in plants, the expression vector pCAMBIA1300-GFP (Fig. 4A) containing the coding sequence of $A h F B A$ was introduced into tobacco (N. tabacum cv. Yunyan 85) via the Agrobacteriummediated leaf disc transformation method to generate transgenic tobacco plants. The empty pCAMBIA1300GFP was transformed into tobacco using the same method as a control. Positive transgenic lines were screened based on kanamycin resistance and confirmed by PCR detection using leaf genomic DNA as a template. The PCR analysis revealed a specific band of approximately $1000 \mathrm{bp}$ in all seven transgenic lines (Fig. 4B) implying that the coding sequence of $A h F B A$ was successfully transformed into tobacco. Furthermore, the transcriptional profiles of AhFBA were detected by real-time qPCR in $\mathrm{T}_{1}$ transgenic lines. As shown in Fig. 4C, the expression of $A h F B A$ varied in seven transgenic lines, whereas the expression was not detected in control plants. Three independent transgenic lines $\left(\mathrm{L}_{2}, \mathrm{~L}_{5}\right.$, and $\left.\mathrm{L}_{6}\right)$ with the highest expressions were selected for further study.

To explore the subcellular localization of AhFBA, the recombinant vectors pCAMBIA1300-AhFBA-GFP and pCAMBIA1300-GFP (as a control) were transiently expressed in tobacco leaves (mediated by Agrobacterium strain LBA 4404). Confocal microscopy analysis indicated that 35S-GFP showed fluorescence signal throughout the whole epidermal cell (Fig. 5A-C), whereas the recombinant protein AhFBA-GFP had a strong
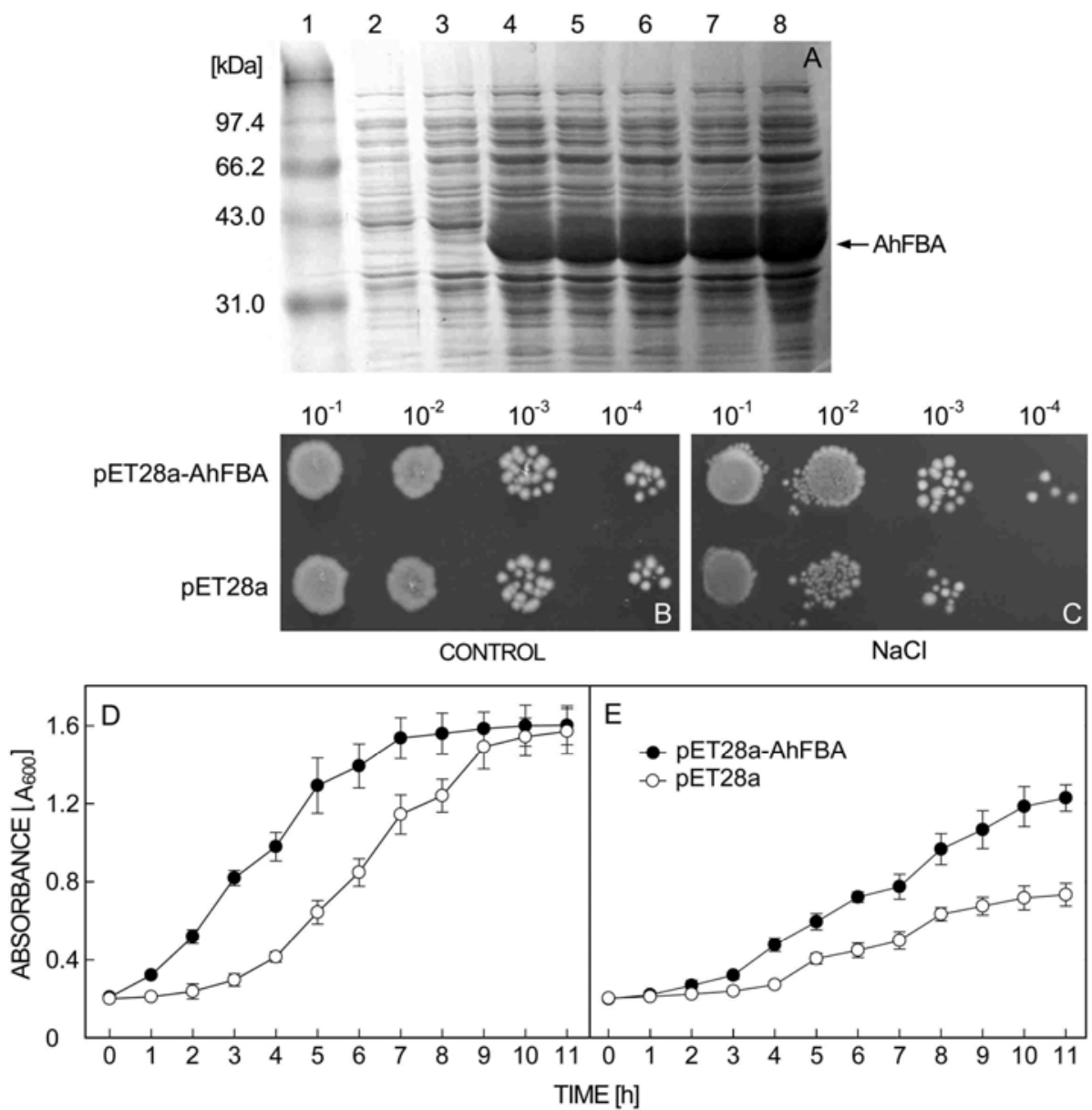

Fig. 3. Analysis by SDS-PAGE of the recombinant protein AhFBA expressed in Escherichia coli strain BL21(DE3) and the role of protein AhFBA in $\mathrm{NaCl}$ stress tolerance of E. coli. A - lane 1 - protein marker; lane 2 - proteins from BL21(DE3) cells harboring a plasmid pET28a induced at $37^{\circ} \mathrm{C}$ with $0.1 \mathrm{mM}$ IPTG for $3 \mathrm{~h}$, lanes 3, 4, 5, 6, 7 and 8 - proteins from BL21(DE3) cells carrying recombinant plasmid pET28a-AhFBA induced at $37^{\circ} \mathrm{C}$ with $0.1 \mathrm{mM}$ IPTG for $0,1,2,3,4$, and 5 h. Proteins were visualized with Coomassie brilliant blue G-250. The arrow indicates the target protein with a molecular mass of approximately $38 \mathrm{kDa}$. Cultures of BL21(DE3) (harbouring pET28a-AhFBA or pET28a) were serially diluted and spotted on a solid LB medium without $(B)$ or with $(C)$ addition of $200 \mathrm{mM} \mathrm{NaCl}$. Growth curves of $E$. coli transformed either with pET28a-AhFBA or with pET28a in a liquid LB medium without $(D)$ and with $(E) 200 \mathrm{mM} \mathrm{NaCl}$. Means \pm SEs, $n=3$. 
cytoplasmic signal in the cells (Fig. 5D-F).

To determine whether the overexpression of protein AhFBA could enhance salinity tolerance, five-week-old tobacco plants were irrigated with $500 \mathrm{mM} \mathrm{NaCl}$ solution every $3^{\text {rd }}$ day, and the growth performance of positive transgenic plants was compared with control plants (Fig. 6). The results revealed no obvious phenotypic differences among control and transgenic tobacco plants grown under normal conditions. However, the control plants showed wilted leaves after $4 \mathrm{~d}$ of $500 \mathrm{mM} \mathrm{NaCl}$ treatment, whereas the transgenic $\mathrm{L}_{2}, \mathrm{~L}_{5}$, and $\mathrm{L}_{6}$ all maintained healthy leaves. Chlorophyll loss and withered and yellow leaves were observed in control plants with the prolongation of salt stress to $7 \mathrm{~d}$, but transgenic lines displayed good growth and less bleaching leaves. The phenotypic characteristics suggest that overexpression of $A h F B A$ enhanced the tolerance of tobacco to salinity.

To explore the effects of $A h F B A$ overexpression on the salinity tolerance of tobacco, some physiological parameters were measured in control and transgenic plants
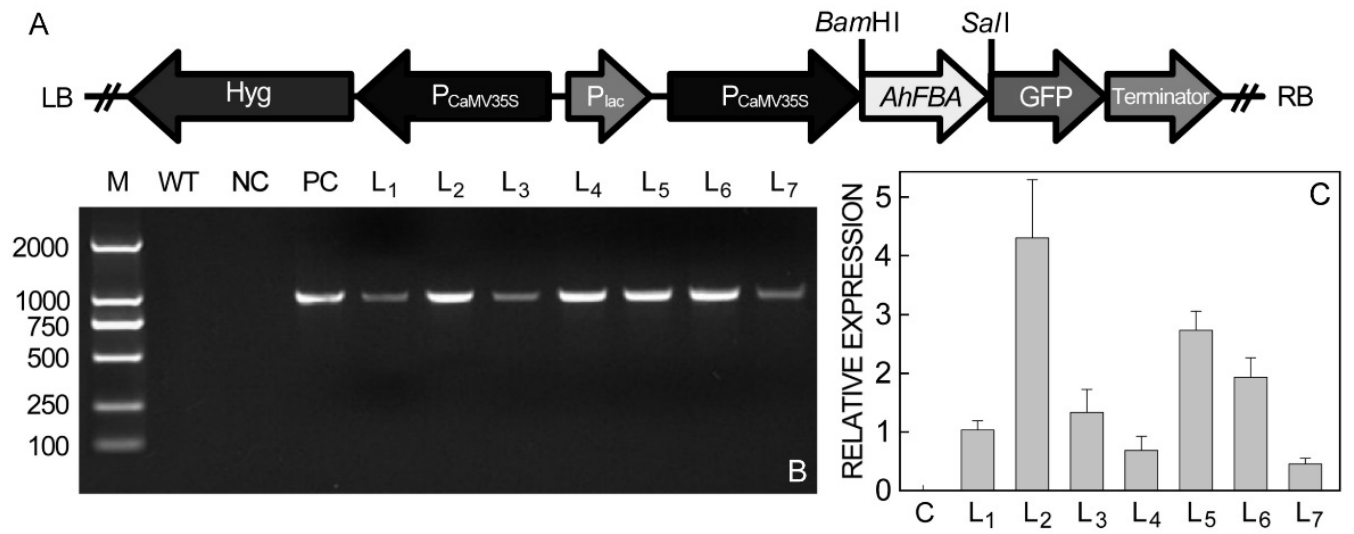

Fig. 4. Generation and detection of transgenic tobacco plants expressing the AhFBA gene. A A schematic diagram of the construction of the recombinant plasmid pCAMBIA1300-AhFBA-GFP. The AhFBA coding sequence was ligated into pCAMBIA1300-GFP under the control of the promoter CaMV 35S. LB - left border, RB - right border. $B$ The PCR amplification of the genomic DNA confirmed the introduction of the AhFBA gene into transgenic tobacco lines. M - DL2000 DNA marker, WT - wild-type, NC - negative control, $\mathrm{PC}$ - positive control, $\mathrm{L}_{1}$ to $\mathrm{L}_{7}$ - independent transgenic tobacco $\mathrm{T}_{1}$ lines overexpressing AhFBA. $C$ - The AhFBA transcript abundance in control and transgenic plants. $\mathrm{C}$ - control; $\mathrm{L}_{1}$ to $\mathrm{L}_{7}$, seven transgenic lines.
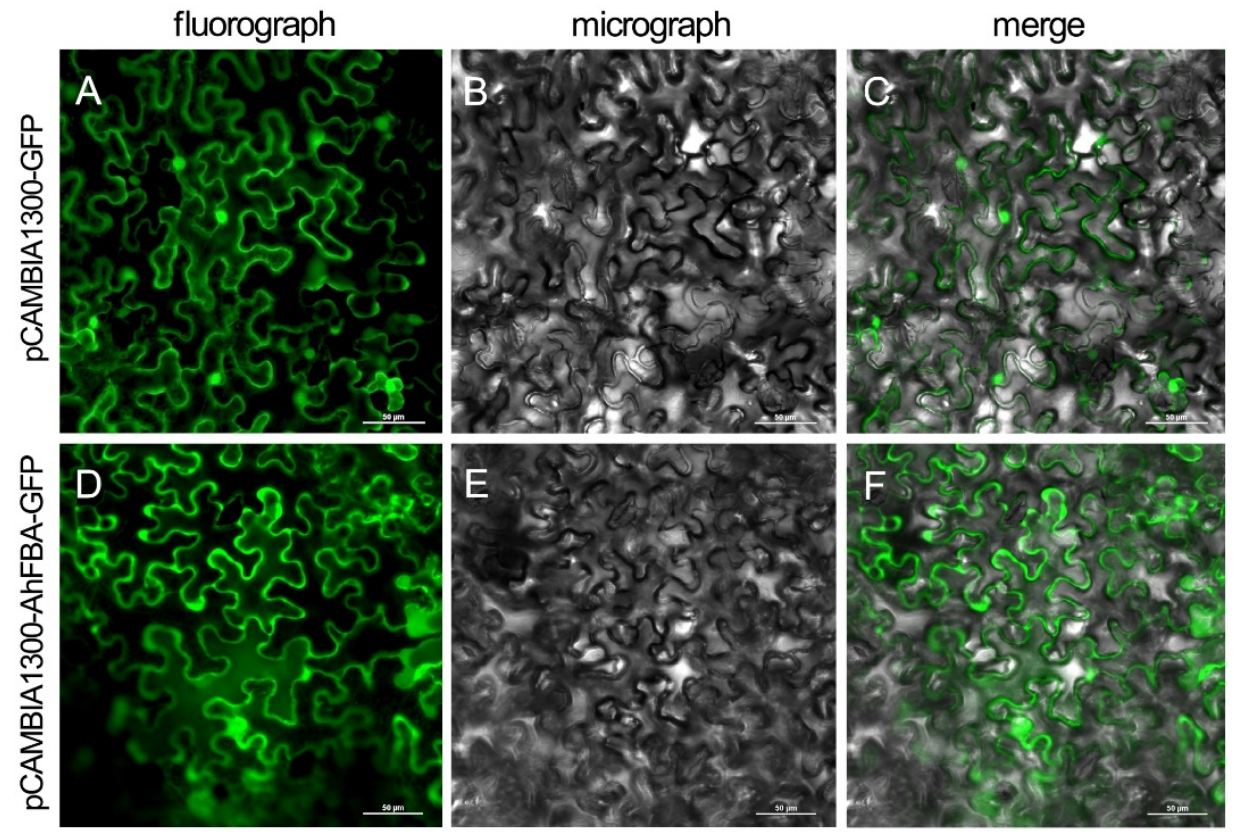

Fig. 5. Subcellular localization of AhFBA in Nicotiana tabacum cv. Yunyan 85. The coding sequence of AhFBA was cloned into the pCAMBIA1300-GFP vector under the control of the CaMV 35S promoter and transformed into leaves of $N$. tabacum. Images were observed using a confocal laser scanning microscope to observe green fluorescence in dark field $48 \mathrm{~h}$ after agroinfiltration $(A, D)$, cell morphology in bright field $(B, E)$, and their combination $(C, F)$. The empty vector (pCAMBIA1300-GFP) was used as a control. 
( $\mathrm{L}_{2}, \mathrm{~L}_{5}$, and $\mathrm{L}_{6}$ ) grown under normal and salt-stressed conditions.

In the absence of $\mathrm{NaCl}(0 \mathrm{~d})$, there were no significant differences in photosynthetic efficiency (the variable to

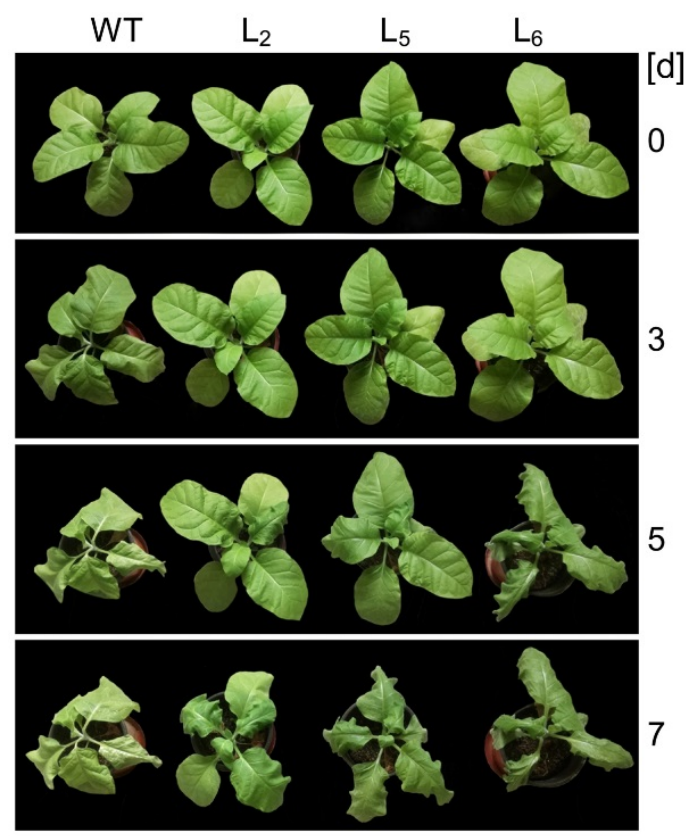

Fig. 6. Growth performances of control (WT) and $A h F B A$ overexpressing $\mathrm{T}_{1}$ transgenic line $\left(\mathrm{L}_{2}, \mathrm{~L}_{5}\right.$, and $\left.\mathrm{L}_{6}\right)$ tobacco plants irrigated with a $500 \mathrm{mM} \mathrm{NaCl}$ solution for 4 and $7 \mathrm{~d}$.

\section{Discussion}

The FBA is a multiple functional protein, which participates in a wide range of biological processes. It is well known that FBA catalyzes the cleavage of FBP into G3P and DHAP and further a reversible aldol condenzation, and it is a vital enzyme in glycolysis, gluconeogenesis, and the Calvin cycle (Haake et al. 1998). Recently, FBA has been shown to participate in other functions in cells, such as the binding of class I cFBA in Medicago sativa to the transcription factor NMH7 (PáezValencia et al. 2008) and the physical interaction of yeast FBA1 with the RNA polymerase III (Pol III) complex (Cieśla et al. 2014).

Although FBA genes have been isolated and studied in numerous plant species, few studies have examined the AhFBA gene in peanut. In this research, the ORF of the AhFBA gene in A. hypogaea was cloned and characterized, and its amino acid sequence showed a high homology with those from $M$. truncatula, $P$. vulgaris, and C. clementina. Sequence alignment indicates that $A h F B A$ was a class-I aldolase. Moreover, subcellular localization demonstrates that $A h F B A$ was located in the cytoplasm. In the present study, overexpression of the protein AhFBA in E. coli and tobacco enhanced salinity tolerance compared with controls in agreement with previously reported studies maximum chlorophyll fluorescence ratio, $\left.\mathrm{F}_{\mathrm{v}} / \mathrm{F}_{\mathrm{m}}\right)$ among control and transgenic plants (Fig. 7A). When subjected to $500 \mathrm{mM} \mathrm{NaCl}$ for 4 and $7 \mathrm{~d}$, the value of $\mathrm{F}_{\mathrm{v}} / \mathrm{F}_{\mathrm{m}}$ were significantly lower in control plants compared with transgenic lines. The $\mathrm{P}_{\mathrm{N}}$ was significantly higher in transgenic lines $\mathrm{L}_{2}$ and $\mathrm{L}_{5}$ than in control plants when exposed to $\mathrm{NaCl}$ treatment for 4 and $7 \mathrm{~d}$ (Fig. 7B). Increased generation of $\mathrm{H}_{2} \mathrm{O}_{2}$ in plants exposed to stresses usually causes a disruption of membrane integrity, which can be also estimated by measuring electrolyte leakage and MDA content in the plants. The results indicate no significant differences in $\mathrm{H}_{2} \mathrm{O}_{2}$ content, electrolyte leakage, and MDA content between control and transgenic plants without salt stress; however, an increase in $\mathrm{H}_{2} \mathrm{O}_{2}$ content, electrolyte leakage, and MDA content were observed in control plants exposed to $500 \mathrm{mM} \mathrm{NaCl}$. Also, RWC was lower in control plants compared with transgenic lines following the application of salt stress (Fig. 7C-F). Sucrose and proline may function as an osmoprotectants maintaining pressure potential, and they also stabilize cellular membranes. Although there were no significant differences in sucrose content between control and transgenic plants under control conditions, all transgenic lines accumulated a significantly higher amount of sucrose than control plants under salinity (Fig. 7G). Similarly, proline content was significantly higher in transgenic lines than in control plants at the fourth day of salinity treatment (Fig. $7 H$ ).

(Fan et al. 2009, Zeng et al. 2015, Cai et al. 2016). Thus, the AhFBA gene could play a positive role in responses to $\mathrm{NaCl}$ stress.

Two isoenzymes of FBA in green plants are cpFBA and cFBA. The former is a significant enzyme in the Calvin cycle in the chloroplast, and the latter is a vital enzyme in glycolytic processes and gluconeogenesis in the cytoplasm (Lebherz et al. 1984). Overexpression of the Solanum lycopersicum cFBA gene in tomato increases the activities of the main enzymes involved in the Calvin cycle and also $\mathrm{P}_{\mathrm{N}}$ (Cai et al. 2016); however, no significant increase in $\mathrm{P}_{\mathrm{N}}$ was observed in transgenic tobacco lines without salinity stress compared with twild type plants (Fig. 7B). Similar results have been obtained by Uematsu et al. (2012), who observed that overexpression of the Arabidopsis thaliana cpFBA gene in tobacco does not enhance photosynthesis in the transgenic plants, particularly at an ambient $\mathrm{CO}_{2}$ concentration. Since the photosynthesis rate is limited by the activity of ribulose1,5-bisphosphate carboxylase/oxygenase at relatively low $\mathrm{CO}_{2}$ concentrations, our results suggest that overexpression of the $C F B A$ gene in tobacco might not increase the activity of ribulose-1,5-bisphosphate carboxylase/oxygenase, and that a native plastid FBA 


\section{Z.K. DU et al.}

activity is sufficient for photosynthesis at ambient $\mathrm{CO}_{2}$ concentrations without environmental stress. Although $\mathrm{P}_{\mathrm{N}}$ of transgenic tobacco lines was notably higher than in control plants under salinity stress for 4 and $7 \mathrm{~d}$, the main explanation might be that photosystem II was less damaged by $\mathrm{NaCl}$ in transgenic plants compared with control plants (Fig. 7A).

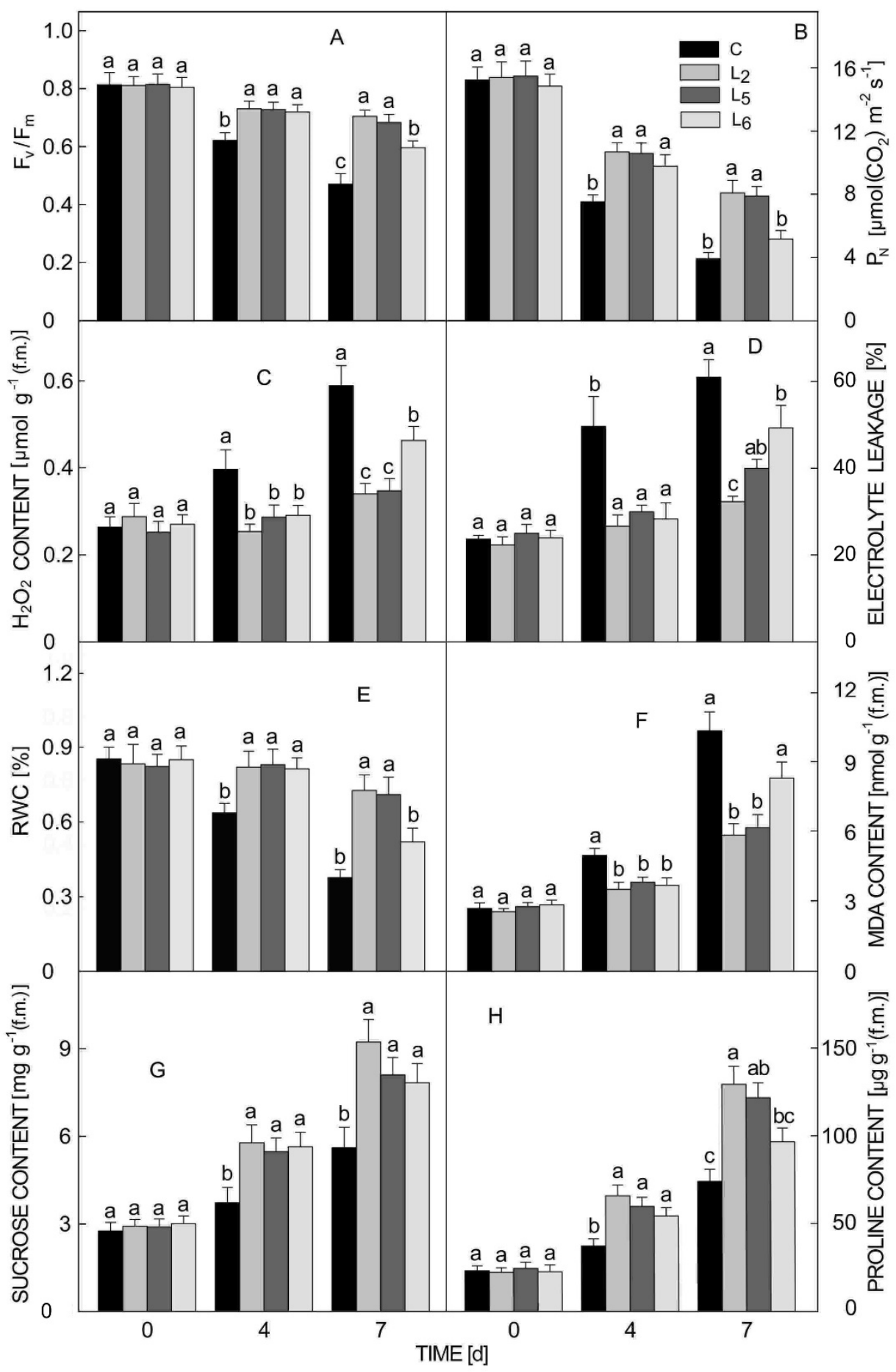

Fig. 7. Physiological analysis of control and transgenic plants $\left(\mathrm{L}_{2}, \mathrm{~L}_{5}\right.$, and $\left.\mathrm{L}_{5}\right)$ grown under $500 \mathrm{mM} \mathrm{NaCl}$ for 0 to $7 \mathrm{~d}$. $A$ - photosynthetic efficiency, $\mathrm{F}_{\mathrm{v}} / \mathrm{F}_{\mathrm{m}} ; B$ - net photosynthetic rate, $\mathrm{P}_{\mathrm{N}} ; C-\mathrm{H}_{2} \mathrm{O}_{2}$ content; $D$ - electrolyte leakage; $E$ - relative water content, RWC; $F$ - malondialdehyde (MDA) content; $G$ - sucrose content; H - proline content. Means \pm SEs from three biological replicates. Different lowercase letters indicate significant differences $(P<0.05)$. 
A major threat to agriculture, especially in arid and semiarid regions, is soil salinization (Hanin et al. 2016). Increased salt accumulation imposes osmotic stress, oxidative stress, ion toxicity, and nutrient deficiency in plants, affecting nearly all aspects of their life cycle, including germination, vegetative growth, and reproductive development (Shrivastava and Kumar 2015). Fortunately, plants have developed two types of osmolytes to avoid the osmotic effects of salt stress, one of which is made up of organic osmolytes, which play an important role in osmotic adjustment. The organic osmolytes commonly found in higher plants consist of sugars and their derivatives, amino acids, and methylated tertiary $\mathrm{N}$ compounds (Chen and Jiang 2010). As a typical osmoprotectant, sucrose is important for maintaining cell osmotic pressure and cell membrane stability (Lee et al. 2013). The cFBA is a key enzyme in the sucrose biosynthetic pathway, where it catalyzes the production of FBP from triose phosphates in the cytoplasm. Transgenic potatoes were generated that express the E. coli $c F B A$ gene to improve plant yield by increasing sucrose production in general; in contrast, decreased cFBPase expression of the CFBA gene in A. thaliana leads to an inhibition of sucrose synthesis (Strand et al. 2000). In the present study, there were no significant differences in sucrose content in leaves among the transgenic lines and control plants (Fig. 7G) without salinity stress, potentially because the synthesized sucrose was rapidly transported to the phloem rather than accumulating in photosynthesizing leaves (Uematsu et al. 2012). However, tobacco overexpressing the AhFBA gene might profit from more sucrose synthesized via the FBAinvolved biosynthesis pathway and stored in leaves when exposed to salt stress, which could promote their water potential under salinity stress.

Proline usually accumulates in a variety of plants in response to biotic and abiotic stresses (Hayat et al. 2012) and it can also be used as an osmolyte to assist plants in adjusting their cellular osmotic potential. In this study, transgenic lines overexpressing the AhFBA gene subjected to salt stress accumulated more proline than control plants suggesting that increased proline might help plants avoid the osmotic stress induced by high salinity. In accordance

\section{Reference}

Abd Elgawad, H., Zinta, G., Hegab, M.M., Pandey, R., Asard, H., Abuelsoud, W.: High salinity induces different oxidative stress and antioxidant responses in maize seedlings organs. Front. Plant Sci. 7: 1-11, 2016.

Cai, B., Li, Q., Xu, Y., Yang, L., Bi, H., Ai, X.: Genome-wide analysis of the fructose 1, 6-bisphosphate aldolase (FBA) gene family and functional characterization of FBA7 in tomato. - Plant Physiol. Biochem. 108: 251-265, 2016.

Chen, H., Jiang, J.-G.: Osmotic adjustment and plant adaptation to environmental changes related to drought and salinity. Environ. Rev. 18: 309-319, 2010.

Chen, Y., Cui, J., Li, G., Yuan, M., Zhang, Z., Yuan, S., Zhang, with this study, there is a strong relationship between the shoot water potential of Reaumuria vermiculata and $\mathrm{NaCl}-$ enriched nutrient solutions, and also proline accumulation in transgenic tobacco shoots significantly increased with increaing $\mathrm{NaCl}$ exposure (Fig. $7 \mathrm{H}$ ), which might play a critical role in osmoregulation.

Apart from causing osmotic stress, high salinity can also trigger the generation of reactive oxygen species (ROS), such as $\mathrm{H}_{2} \mathrm{O}_{2}$, within plant cells, which (at a high level) results in the oxidative damage of membrane lipids, sugars, proteins, and nucleic acids (AbdElgawad et al. 2016). To cope with the oxidative stress resulting from ROS, a complex scavenging system consisting of enzymatic and nonenzymatic antioxidants has been evolved in higher plants. In addition to acting as an osmolyte, proline can also be considered an antioxidant, which contributes to ROS scavenging (Wu et al. 2014). The application of exogenous proline drastically reduces the oxidative damage to lipid membranes in tobacco caused by salinity (Okuma et al. 2004). In the present study, control plants accumulated 2.33-fold more $\mathrm{H}_{2} \mathrm{O}_{2}$ under salt stress for $7 \mathrm{~d}$. However, only a slight increase in $\mathrm{H}_{2} \mathrm{O}_{2}$ content was detected in transgenic tobacco plants (Fig. 7C). This observation imply that overexpression of the AhFBA gene either inhibited $\mathrm{H}_{2} \mathrm{O}_{2}$ generation or effectively scavenged excess $\mathrm{H}_{2} \mathrm{O}_{2}$ by proline.

In this study, the AhFBA gene was cloned from A. hypogaea and functionally characterized. Bioinformatic analysis reveals that AhFBA belonged to the class-I aldolases, and in silico and in vivo localization studies suggest that the AhFBA protein was localized in the cytoplasm. Prokaryotic expression revealed notably enhanced salinity tolerance and survival of $E$. coli. Transgenic tobacco overexpressing the AhFBA gene exhibited a lower $\mathrm{H}_{2} \mathrm{O}_{2}$ content, electrolyte leakage, and malondialdehyde content and a higher photosynthetic efficiency, $\mathrm{P}_{\mathrm{N}}$, relative water content, sucrose content, and proline content, compared to control plants. Taken together, the results indicate that overexpression of AhFBA increased the tolerance of $E$. coli and $N$. tabacum to salinity stress, probably by scavenging $\mathrm{H}_{2} \mathrm{O}_{2}$ and maintaining the osmotic balance.
H.: Effect of salicylic acid on the antioxidant system and photosystem II in wheat seedlings. - Biol. Plant. 60: 139-147, 2016.

Cheng, L., Wang, Y., He, Q., Li, H., Zhang, X., Zhang, F.: Comparative proteomics illustrates the complexity of drought resistance mechanisms in two wheat (Triticum aestivum L.) cultivars under dehydration and rehydration. BMC Plant Biol. 16: 188-210, 2016.

Cieśla, M., Mierzejewska, J., Adamczyk, M., Farrants, A.-K.Ö., Boguta, M.: Fructose bisphosphate aldolase is involved in the control of RNA polymerase III-directed transcription. - BBA 1843: 1103-1110, 2014. 
Dennis, E., Gerlach, W., Walker, J., Lavin, M., Peacock, W. Anaerobically regulated aldolase gene of maize:a chimaeric origin? - J. mol. Biol. 202: 759-767, 1988.

Fan, W., Zhang, Z., Zhang, Y.: Cloning and molecular characterization of fructose-1,6-bisphosphate aldolase gene regulated by high-salinity and drought in Sesuvium portulacastrum. - Plant Cell Rep. 28: 975-984, 2009.

Flechner, A., Gross, W., Martin, W.F., Schnarrenberger, C.: Chloroplast class I and class II aldolases are bifunctional for fructose-1, 6-biphosphate and sedoheptulose-1,7biphosphate cleavage in the Calvin cycle. - FEBS Lett. 447: 200-202, 1999.

Gross, W., Lenze, D., Nowitzki, U., Weiske, J., Schnarrenberger, C.: Characterization, cloning, and evolutionary history of the chloroplast and cytosolic class I aldolases of the red alga Galdieria sulphuraria. - Gene 230: 7-14, 1999.

Haake, V., Zrenner, R., Sonnewald, U., Stitt, M.: A moderate decrease of plastid aldolase activity inhibits photosynthesis, alters the levels of sugars and starch, and inhibits growth of potato plants. - Plant J. 14: 147-157, 1998.

Hanin, M., Ebel, C., Ngom, M., Laplaze, L., Masmoudi, K.: New insights on plant salt tolerance mechanisms and their potential use for breeding. - Front. Plant Sci. 7: 1-17, 2016.

Hayat, S., Hayat, Q., Alyemeni, M.N., Wani, A.S., Pichtel, J., Ahmad, A.: Role of proline under changing environments: a review. - Plant Signal. Behav. 7: 1456-1466, 2012.

Kagaya, Y., Nakamura, H., Ejiri, S.-i., Tsutsumi, K.-i., Hidaka, S.: The promoter from the rice nuclear gene encoding chloroplast aldolase confers mesophyll-specific and lightregulated expression in transgenic tobacco. - Mol. gen. Genet. 248: 668-674, 1995.

Kavas, M., Akça, O.E., Akçay, U.C., Peksel, B., Eroğlu, S., Öktem, H.A., Yücel, M.: Antioxidant responses of peanut (Arachis hypogaea L.) seedlings to prolonged salt-induced stress. - Arch. Biol. Sci. 67: 1303-1312, 2015.

Konishi, H., Yamane, H., Maeshima, M., Komatsu, S.: Characterization of fructose-bisphosphate aldolase regulated by gibberellin in roots of rice seedling. - Plant mol. Biol. 56: 839-848, 2004

Lao, X., Azuma, J.-i., Sakamoto, M.: Two cytosolic aldolases show different expression patterns during shoot elongation in moso bamboo, Phyllostachys pubescens Mazel. - Physiol. Plant. 149: 422-431, 2013.

Lebherz, H., Leadbetter, M., Bradshaw, R.: Isolation and characterization of the cytosolic and chloroplast forms of spinach leaf fructose diphosphate aldolase. - J. biol. Chem. 259: 1011-1017, 1984.

Lee, B.-R., Muneer, S., Park, S.-H., Zhang, Q., Kim, T.-H.: Ammonium-induced proline and sucrose accumulation, and their significance in antioxidative activity and osmotic adjustment. - Acta Physiol. Plant. 35: 2655-2664, 2013.

Lv, G.Y., Guo, X.G., Xie, L.P., Xie, C.G., Zhang, X.H., Yang, Y., Xiao, L., Tang, Y.Y., Pan, X.L., Guo, A.G.: Molecular characterization, gene evolution, and expression analysis of the fructose-1,6-bisphosphate aldolase (FBA) gene family in wheat (Triticum aestivum L.). - Front. Plant Sci. 8: 1-18, 2017.

Mohapatra, S., Mittra, B.: Protein glutathionylation protects wheat (Triticum aestivum var. Sonalika) against Fusarium induced oxidative stress. - Plant Physiol. Biochem. 109: 319$325,2016$.

Okuma, E., Murakami, Y., Shimoishi, Y., Tada, M., Murata, Y.: Effects of exogenous application of proline and betaine on the growth of tobacco cultured cells under saline conditions. - Soil Sci. Plant Nutr. 50: 1301-1305, 2004.

Páez-Valencia, J., Valencia-Mayoral, P., Sánchez-Gómez, C., Contreras-Ramos, A., Hernández-Lucas, I., MartínezBarajas, E., Gamboa-deBuen, A.: Identification of fructose1,6-bisphosphate aldolase cytosolic class I as an NMH7 MADS domain associated protein. - Biochem. biophys. Res. Commun. 376: 700-705, 2008.

Park, J., Tran, L. H., Jung, S.: A protoporphyrinogen oxidase gene expression influences responses of transgenic rice to oxyfluorfen. - Biol. Plant. 61: 659-666, 2017.

Patel, M. K., Joshi, M., Mishra, A., Jha, B.: Ectopic expression of SbNHX1 gene in transgenic castor (Ricinus communis L.) enhances salt stress by modulating physiological process. Plant Cell Tissue Organ Cult. 122: 477-490, 2015.

Purev, M., Kim, M.K., Samdan, N., Yang, D.-C.: Isolation of a novel fructose-1,6-bisphosphate aldolase gene from Codonopsis lanceolata and analysis of the response of this gene to abiotic stresses. - Mol. Biol. 42: 179-186, 2008.

Schmittgen, T.D., Livak, K.J.: Analyzing real-time PCR data by the comparative CT method. - Nat. Protoc. 3: 1101-1108, 2008.

Shams, F., Oldfield, N.J., Wooldridge, K.G., Turner, D.P.: Fructose-1,6-bisphosphate aldolase (FBA) - a conserved glycolytic enzyme with virulence functions in bacteria: 'ill met by moonlight'. - Biochem. Soc. Trans. 42: 1792-1795, 2014.

Shi, H., Wang, Y., Cheng, Z., Ye, T., Chan, Z.: Analysis of natural variation in bermudagrass (Cynodon dactylon) reveals physiological responses underlying drought tolerance. - PLoS ONE 7: e53422, 2012.

Shrivastava, P., Kumar, R.: Soil salinity: a serious environmental issue and plant growth promoting bacteria as one of the tools for its alleviation. - Saudi J. biol. Sci. 22: 123-131, 2015.

Singh, P.K., Shrivastava, A.K., Chatterjee, A., Pandey, S., Rai, S., Singh, S., Rai, L.: Cadmium toxicity in diazotrophic Anabaena spp. adjudged by hasty up-accumulation of transporter and signaling and severe down-accumulation of nitrogen metabolism proteins. - J. Proteomics 127: 134-146, 2015.

Strand, Å., Zrenner, R., Trevanion, S., Stitt, M., Gustafsson, P., Gardeström, P.: Decreased expression of two key enzymes in the sucrose biosynthesis pathway, cytosolic fructose-1,6bisphosphatase and sucrose phosphate synthase, has remarkably different consequences for photosynthetic carbon metabolism in transgenic Arabidopsis thaliana. - Plant J. 23: 759-770, 2000.

Sui, J., Jiang, D., Zhang, D., Song, X., Wang, J., Zhao, M., Qiao, L.: The salinity responsive mechanism of a hydroxyprolinetolerant mutant of peanut based on digital gene expression profiling analysis. - PLoS ONE 11: e0162556, 2016.

Toscano, S., Farieri, E., Ferrante, A., Romano, D.: Physiological and biochemical responses in two ornamental shrubs to drought stress. - Front. Plant Sci. 7: ??-??, 2016.

Uematsu, K., Suzuki, N., Iwamae, T., Inui, M., Yukawa, H.: Increased fructose 1,6-bisphosphate aldolase in plastids enhances growth and photosynthesis of tobacco plants. - J. exp. Bot. 63: 3001-3009, 2012.

Wu, G., Wang, G., Ji, J., Gao, H., Guan, W., Wu, J., Guan, C., Wang, Y.: Cloning of a cytosolic ascorbate peroxidase gene from Lycium chinense Mill. and enhanced salt tolerance by overexpressing in tobacco. - Gene 543: 85-92, 2014.

Zeng, Y., Tan, X., Zhang, L., Jiang, N., Cao, H.: Identification 
and expression of fructose-1,6-bisphosphate aldolase genes and their relations to oil content in developing seeds of tea oil tree (Camellia oleifera). - PLoS ONE 9: e107422, 2014.

Zeng, Y., Tan, X., Zhang, L., Long, H., Wang, B., Li, Z., Yuan, Z.: A fructose-1, 6-biphosphate aldolase gene from Camellia oleifera: molecular characterization and impact on salt stress tolerance. - Mol. Breed. 35: 17, 2015.

Zhang, F., Li, S., Yang, S., Wang, L., Guo, W.: Overexpression of a cotton annexin gene, GhAnn1, enhances drought and salt stress tolerance in transgenic cotton. - Plant mol. Biol. 87: 4767, 2015.

Zhang, F., Zhang, P., Zhang, Y., Wang, S., Qu, L., Liu, X., Luo, J.: Identification of a peroxisomal-targeted aldolase involved in chlorophyll biosynthesis and sugar metabolism in rice. Plant Sci. 250: 205-215, 2016. 